\section{Integrins' prime syndecan}

\author{
By Michael J. Haas, Senior Writer
}

Researchers at the University of Wisconsin-Madison have shown that synstatin, a peptide derived from syndecan-1, can disrupt the interaction between the parent molecule and integrin $\alpha_{v} \beta_{3}$ or integrin $\alpha_{\mathrm{v}} \beta_{5}$, thus preventing angiogenesis and decreasing tumor growth in mice. ${ }^{1}$ The findings suggest that the cross talk between syndecan-1 and integrins could be a critical component of tumor angiogenesis. If the results translate to human cancers, synstatin or a mimetic may offer an alternative to integrin inhibitors in the clinic.

Integrins are a class of extracellular signaling molecules that regulate cell morphology and mobility, growth factor signaling and the cell cycle. They are composed of differing $\alpha$ and $\beta$ subunit chains. Integrins containing the $\alpha_{v}$ subunit are expressed in many cancers, and integrins $\alpha_{v} \beta_{3}$ and $\alpha_{v} \beta_{5}$ are known to interact with VEGF and fibroblast growth factor (FGF) during tumor angiogenesis. At least five companies are developing $\alpha_{\mathrm{v}}$ integrin inhibitors to treat cancer (see Box 1, "Turn of cilengitide").

Syndecans are a family of heparan sulfate proteoglycan cell-surface receptors that regulate cell adhesion, migration, growth and development by interacting with growth factors and extracellular matrix proteins. Aberrant expression of syndecan-1 (SDC1; CD138) has been implicated in cancer. ${ }^{2}$

The Wisconsin-Madison team had already shown in a 2004 study that a soluble, 252-amino-acid form of the SDC1 ectodomain disrupted the SDC1-integrin $\alpha_{\mathrm{v}} \beta_{3}$ interaction and prevented human breast cancer cells from spreading in vitro. ${ }^{3}$

In a new study in the Journal of Experimental Medicine, the team looked at whether a shorter version of the SDC1 ectodomain would have a similar effect on normal endothelial cells, which a nascent tumor must induce to build blood vessels so the tumor can grow and survive. Moreover, because there is little angiogenesis in normal adults, targeting these cells would not be expected to have a negative side effect.

Indeed, the 32-residue peptide synstatin, which was derived from the SDC1 backbone, prevented human aortic and microvascular endothelial cells from spreading in vitro.

The molecule competed with cell-surface SDC1 to block integrin $\alpha_{v} \beta_{3}$ activation and inhibit the in vitro spread of human mammary cancer cells. Subcutaneous synstatin also decreased tumor volume compared with that seen in mouse models of mammary carcinoma. Tumors from the treated mice had 11-fold less growth of new blood vessels than tumors from control animals.
The team was led by Alan Rapraeger, professor of pathology and laboratory medicine at Wisconsin-Madison.

\section{Synstatin's prime scene}

Although SDC1-integrin interactions clearly play a role in animal models, it is not clear whether those interactions occur in human tumors. In addition, SDC1 might not be a lynchpin in the angiogenesis process.

"The initial findings demonstrating activity of synstatin in a tumor xenograft mouse model are suggestive" of relevance to human tumor angiogenesis, said Thomas Chittenden, executive director of research at ImmunoGen Inc. But he suggested that delayed tumor growth in response to treatment "may result from a direct effect of the peptide on the tumor cells - which also express SDC1 and integrins"-rather than inhibition of angiogenesis in normal cells.

He also said the results in the JEM article showed that synstatin did not prevent the spread of human umbilical vein endothelial cells in vitro or prevent angiogenesis in mice that did not express SDC1. Based on those data, the study authors concluded that SDC1-deficient cells use compensatory mechanisms for angiogenesis.

"This raises questions about whether SDC1 has a pivotal role in angiogenesis," Chittenden said. "Such compensatory mechanisms could operate in tumor cells and/or tumor blood vessels and thereby limit the effectiveness of synstatin or similar agents."

ImmunoGen develops antibody-drug conjugates to treat cancer. The company's IMGN 388 is in Phase I testing to treat solid tumors. The compound combines ImmunoGen's DM4 cell-killing agent and an antibody targeting an undisclosed integrin. The antibody was developed at the Centocor Inc. unit of Johnson \& Johnson. ImmunoGen received exclusive worldwide rights to the compound in a 2008 deal.

Because SDC1-deficient cells have alternate routes to angiogenesis, "resistance to synstatin may develop during therapy," said Martin Götte, research laboratory head in gynecology and obstetrics at The University Hospital of Muenster.

However, because SDC1 and $\alpha_{\mathrm{v}}$ integrins have been implicated in a wide variety of cancers, synstatin could still be an important component of combination therapies, he said.

"More importantly, SDC1 and these integrins are expressed in blood vessels and are involved in the process of normal angiogenesis," so targeting the interaction in endothelial cells could be a feasible strategy to treat tumors that do not themselves express either SDC1 or $\alpha_{v}$ integrins, Götte said.

Götte added: "The functions of SDC1 in cancer are diverse and sometimes hard to predict." As an example, he pointed to a study his group published this year showing that the soluble form of SDC1 played a different role than the membrane-bound form in breast cancer cells in vitro: the latter suppressed cell proliferation whereas the former promoted cell invasiveness. ${ }^{4}$

Simon Goodman also noted that SDC1's functions are not fully understood. "There is a complex interplay between its membrane-bound form and soluble form, and there are even reports of syndecan-1 finding its way to the nucleus," he said. Goodman is senior scientist of biochemistry 
A study in Nature Medicine suggests that the crop of $\alpha_{v}$ integrin inhibitors currently in development-particularly Merck KGaA's cilengitide-may have undesired dose-dependent side effects that may call their use into question. ${ }^{5}$ Synstatin, one of the first in a class of compounds that block angiogenesis via a different mechanism, may offer a better alternative.

Cilengitide blocks tumor angiogenesis by mimicking the arginine-glycineaspartic acid (RGD) sequence that integrins recognize and bind. Although the compound is usually dosed in the micromolar range, the Nature Medicine study shows that at nanomolar plasma concentrations, cilengitide and another RGD mimetic could actually induce angiogenesis.

Cilengitide is in Phase III testing to treat glioblastoma and Phase I/II trials to treat metastatic squamous cell carcinoma of the head and neck (SCCHN) and advanced non-small cell lung cancer (NSCLC).

Martin Picard, medical leader in Merck KGaA's global clinical development unit for oncology, noted that cilengitide is being tested in combination with radio- or chemotherapy - scenarios not investigated in the Nature Medicine article. The company declined further comment.
The team reporting in Nature Medicine was led by Kairbaan Hodivala-Dilke, principal investigator of the angiogenesis laboratory at Queen Mary, University of London's Barts and The London School of Medicine and Dentistry, and by Andrew Reynolds, staff scientist at The Institute of Cancer Research. The group also included scientists from The Beatson Institute for Cancer Research, Cancer Research UK and Servier.

Hodivala-Dilke said that with a better understanding of the in vivo mechanisms of $\alpha_{v} \beta_{3}$ inhibition, "we will be able to design agents that more efficiently inhibit angiogenesis in the clinical setting."

Martin Götte, research laboratory head in gynecology and obstetrics at The University Hospital of Muenster, noted that clinical trials of RGD-mimetic integrin inhibitors "have been largely unsuccessful, possibly due to a stimulatory effect of these inhibitors on VEGF-mediated angiogenesis, as suggested" by the Nature Medicine study.

Götte added that synstatin, which is derived from syndecan-1 (SDC1; CD138), might have an advantage over RGD mimetics because "SDC1 modulates growth factors, angiogenic factors and chemokine functions" and thus might block VEGF-mediated angiogenesis.

"But whether some of these SDC1 functions are altered by synstatin remains to be shown," he added.

Alan Rapraeger, professor of pathology and laboratory medicine at the University of Wisconsin-Madison, also told SciBX that synstatin might be more effective at blocking angiogenesis than other inhibitors because the peptide targets "an early, fundamental step of the angiogenic signaling mechanism, rather than inhibiting individual integrins or individual signaling pathways after they have been turned on."

Finally, Thomas Chittenden, executive director of research at ImmunoGen Inc. said that "additional in vivo studies could further validate the role of SDC1 in angiogenesis and potentially help develop a rationale for why synstatin might be more effective than existing agents that target integrins."

Hodivala-Dilke said her group is testing whether other inhibitors with higher affinities for integrin $\alpha_{v} \beta_{3}$, or that inhibit it by a non-RGD-mimetic mechanism, can block angiogenesis at low nanomolar concentrations. She said the findings reported in Nature Medicine are not patented. $\quad-\mathrm{MJH}$ and cellular pharmacology in oncology R\&D at Merck KGaA's Merck Serono S.A. unit.

To better understand the potential therapeutic effects of synstatin on tumors, Götte said, the peptide should be studied in nonangiogenic models of cancer, such as those modeling metastasis.

Moreover, although Rapraeger's team showed that SDC1 regulated $\alpha_{\mathrm{v}}$ activity on normal endothelial cells, whether this also occurred during tumor angiogenesis in humans-and thus whether inhibiting that interaction would provide any therapeutic benefit-was not yet clear, said Goodman.

Thus, both Goodman and Martin Picard said additional studies need to investigate the physiological and pathophysiological roles of SDC1 in other signaling pathways. Picard is medical leader of Merck KGaA's global clinical development unit for oncology.

Like Götte, Picard noted that the results in the JEM article in SDC1deficient cells suggested that resistance could develop to synstatin. "It would be desirable to understand these other mechanisms more deeply before any attempt is made to use the peptide or a drug based on it as a therapeutic", he said.
Merck KGaA's cilengitide, a peptide inhibitor of integrins $\alpha_{\mathrm{v}} \beta_{3}$ and $\alpha_{\mathrm{v}} \beta_{5}$, is in Phase III testing to treat glioblastoma and in Phase I/II trials to treat metastatic squamous cell carcinoma of the head and neck (SCCHN) and advanced non-small cell lung cancer (NSCLC).

Rapraeger said his group has unpublished data showing that synstatin prevents melanoma and myeloma cells from spreading in vitro but has not yet determined whether the SDC1-integrin mechanism of angiogenesis occurs in other cancers. His group is in the early stages of developing synstatin mimetics, he said.

The Wisconsin Alumni Research Foundation has filed a patent for synstatin and "is entertaining out-licensing opportunities," he said.

Haas, M.J. SciBX 2(12); doi:10.1038/scibx.2009.473

Published online March 26, 2009

\section{REFERENCES}

1. Beauvais, D. et al. J. Exp. Med.; published online March 2, 2009;

doi:10.1084/jem.20081278

Contact: Alan C. Rapraeger, University of Wisconsin-Madison,

Madison, Wis.

e-mail: acraprae@wisc.edu

2. Yip, W. et al. Mol. Cancer Ther. 5, 2139-2148 (2006) 
3. Beauvais, D. et al. J. Cell Biol. 167, 171-181 (2004)

4. Nikolova, V. et al. Carcinogenesis 30, 397-407 (2009)

5. Reynolds, A. et al. Nat. Med.; published online March 22, 2009; doi:10.1038/nm.1941

Contact: Kairbaan Hodivala-Dilke, The Institute of Cancer Research, London, U.K.

e-mail: Kairbaan.Hodivala-Dilke@cancer.org.uk

Contact: Andrew Reynolds, same affiliation as above

e-mail: andrew.reynolds@icr.ac.uk

COMPANIES AND INSTITUTIONS MENTIONED

The Beatson Institute for Cancer Research, Glasgow, U.K.
Cancer Research UK, London, U.K.

Centocor Inc. (NASDAQ:CNTO), Malvern, $\mathrm{Pa}$.

ImmunoGen Inc. (NASDAQ:IMGN), Waltham, Mass.

The Institute of Cancer Research, London, U.K.

Johnson \& Johnson (NYSE:JNJ), New Brunswick, N.J.

Merck KGaA (Xetra:MRK), Darmstadt, Germany

Merck Serono S.A., Geneva, Switzerland

Queen Mary, University of London, London, U.K.

Servier, Neuilly-sur-Seine, France

The University Hospital of Muenster, Muenster, Germany

University of Wisconsin-Madison, Madison, Wis.

Wisconsin Alumni Research Foundation, Madison, Wis. 